\title{
Optical Recording of Odor-Evoked Responses in the Olfactory Brain of the Naiive and Aversively Trained Terrestrial Snails
}

\author{
Eugeny S. Nikitin and Pavel M. Balaban ${ }^{1}$ \\ Institute of Higher Nervous Activity and Neurophysiology RAS, Moscow 117865, Russia
}

\begin{abstract}
Regular spontaneous oscillations were recorded both electro- and optophysiologically using a voltage-sensitive absorption dye in the olfactory part of the brain (procerebral lobe of the cerebral ganglia) of the gastropod mollusk Helix lucorum. Odor application caused transient changes in procerebral oscillations, and an odor-evoked potential was recorded in the procerebrum (PC). The wave of evoked potential originated near the place of olfactory nerve entrance into the PC and propagated via the procerebral neuropile toward the cell body layer. The spread of the odor-evoked potential corresponded roughly to the neuropile area, whereas the spontaneous oscillations were recorded in the cell body layer of the PC and were not observed in the neuropile. Evoked potential did not produce additional events intercalated into the ongoing spontaneous oscillations. Changes in parameters of spontaneous oscillations to the repeated presentations of the same odor were variable. To estimate the role of spontaneous oscillations in odor encoding, we trained the snail to avoid cineole, using paired presentations of cineole and electric shock. Elaboration of conditioned aversion to cineole applications resulted in distinct pairing-specific changes in behavior of the snails and procerebral activity. Responses to odor (cineole) applications were not different in amplitude or frequency of spontaneous oscillations in control and trained snails, whereas ratio of amplitudes of the same oscillation wave in proximal and distal regions of the procerebrum was significantly different in control and aversively trained snails, reflecting changes in neural firing in certain areas of the olfactory lobe.
\end{abstract}

Olfaction in terrestrial gastropod mollusks (snails and slugs) is an important sensory modality for locating and differentiating odors during feeding and orientation. Mollusks learn a variety of new odors. The acquisition of olfactory memory in Helix require the olfactory stimulation of the sensory epithelia on the anterior tentacles, whereas the recall of memory needs posterior tentacles to be intact (Friedrich and Teyke 1998). Pairing electric shock with food results in a strong aversion to the taste and smell of the conditioned kind of food in snail Helix (Balaban 1993). Likewise, Limax tends to avoid an area with a strong concentration of the odor that previously had been paired with noxious quinidine sulfate (Sahley et al. 1981).

The procerebral lobe (PC) of cerebral ganglia in terrestrial snails is directly involved in olfactory information processing (Gelperin and Tank 1990; Chase and Tolloczko 1993). The PC constitutes the most dorsal part of the cerebral (supraesophageal) ganglion of the snail's nerve ring. Two major parts can be distinguished in the PC: a neuropile and the cell body layer. The neuropile occupies the medial and core position, while the cell body layer joins the neuropile at the lateral side (Zaitseva 1991; Chase and Tolloc-

${ }^{1}$ Corresponding author.

E-MAIL balaban@ihna.msk.ru; FAX 1-617-6873051.

Article and publication are at www.learnmem.org/cgi/doi/ $10.1101 / \mathrm{lm} .32500$. zko 1993). The borders of the cell body layer partially surround the neuropile on the ventral and dorsal sides. A transverse section of the procerebral cell body layer shows a half-moon shape, whereas the neuropile is nested (Ermentrout et al. 1998). The PC of the terrestrial mollusk contains up to $80 \%$ of all the neurons of the central nervous system (CNS). The procerebral neurons are the smallest in the entire nervous system $(5-7 \mu \mathrm{m})$. They are mostly monopolar, sending the neurites into the neuropile of PC (Zaitseva 1991; Chase and Tolloczko 1993).

The spontaneous or stimulus-induced oscillations are common in olfactory systems of both vertebrates and invertebrates. Odor-evoked $20-40-\mathrm{Hz}$ oscillations have been found in mushroom bodies and antennal lobes of insects (Laurent and Davidowitz 1994). Moreover, Wehr and Laurent (1996) demonstrated that odor attributes may be coded in the temporal sequences of firing in oscillating neuronal assemblies of the antennal lobe. Oscillatory olfactory neurons were described in the protocerebrum of the crayfish (Mellon et al. 1992). Oscillations were recorded optically in the olfactory lobe of turtles. Each inspiration of a mammal is followed by several cycles of high-frequency $(60-90 \mathrm{~Hz})$ oscillations of a local field potential in the granular cell layer of the olfactory bulb (Gray and Skinner 1988).

Low-frequency $(\sim 1 \mathrm{~Hz})$ spontaneous oscillations of the local field potential have been described in the PC of the

LEARNING \& MEMORY 7:422-432 @ 2000 by Cold Spring Harbor Laboratory Press ISSN1072-0502/00 \$5.00

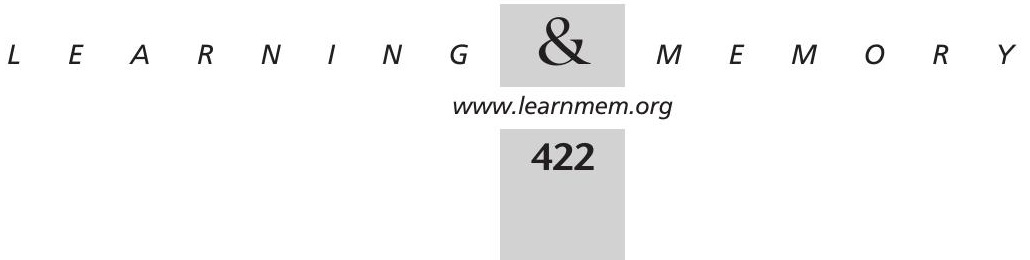


slug Limax maximus (Gelperin and Tank 1990). The waves of activity propagate across the PC from the apical end toward the base. The front of the wave is presented by a linear zone of depolarization followed by a larger and higher-amplitude zone of hyperpolarization. The propagation velocity is $\sim 1.1 \mathrm{~mm} / \mathrm{sec}$ (Delaney et al. 1994). It was shown that odor application causes the electrical activity in the procerebral lobe of the slug Limax to switch transiently from the state of propagating waves to a state with no or only a small phase difference along the lobe (Delaney et al. 1994). Application of different odors in different concentrations induces transient changes in PC oscillations that differ remarkably from each other (Gervais et al. 1996).

In this work, we analyzed responses to odor application in the PC of the terrestrial snail Helix, using absorption voltage-sensitive dyes and a 124-photodiode array for optical recording of electrical events. To estimate the role of spontaneous oscillations in odor encoding, we trained the snails to avoid the odor cineole using paired presentations of cineole and electric shock. Differences in parameters of the PC activity in two groups of snails subjected to explicitly unpaired and paired procedures reflected changes in neural firing in certain areas of the olfactory lobe.

\section{RESULTS}

\section{Spontaneous Oscillations and Evoked Potentials}

Using suction glass electrodes, we recorded spontaneous $0.6-0.8-\mathrm{Hz}$ oscillations of the local field potential in the Helix PC (Fig. 1A). Spontaneous oscillations showed high regularity over more than $1 \mathrm{~h}$ ( $n=9$ preparations). The frequency of oscillations tended to decrease in long-lasting (hours) experiments, and periods of absence of oscillations were also noted.

The oscillations in the PC of Helix were also recorded optically using the voltage-sensitive dye RH155 (Fig. 1B, top trace). The average frequency of oscillations in stained preparations was lower $(0.3-0.7 \mathrm{~Hz})$ than in the unstained ones. It is known that the voltage-sensitive dye may hyperpolarize neurons, and this may cause the difference in frequency. Tracing the maximum of the peak in each detector in 16 experiments, we found that oscillations propagate from the apex of the PC toward its base. The calculated propagation velocity was $2.7 \pm 0.3 \mathrm{~mm} / \mathrm{sec}(n=16$ experiments). The velocity was calculated using the shift between waves on adjacent detectors taken from the shift-predictor cross-corellogramms (Steinmetz et al. 2000). The experiments carried out in the PC of the slug Deroceras (five animals) showed that the shapes of procerebral oscillatory waves in snail Helix and slug Deroceras were generally similar, but they clearly differed in the ratio of the depolar-
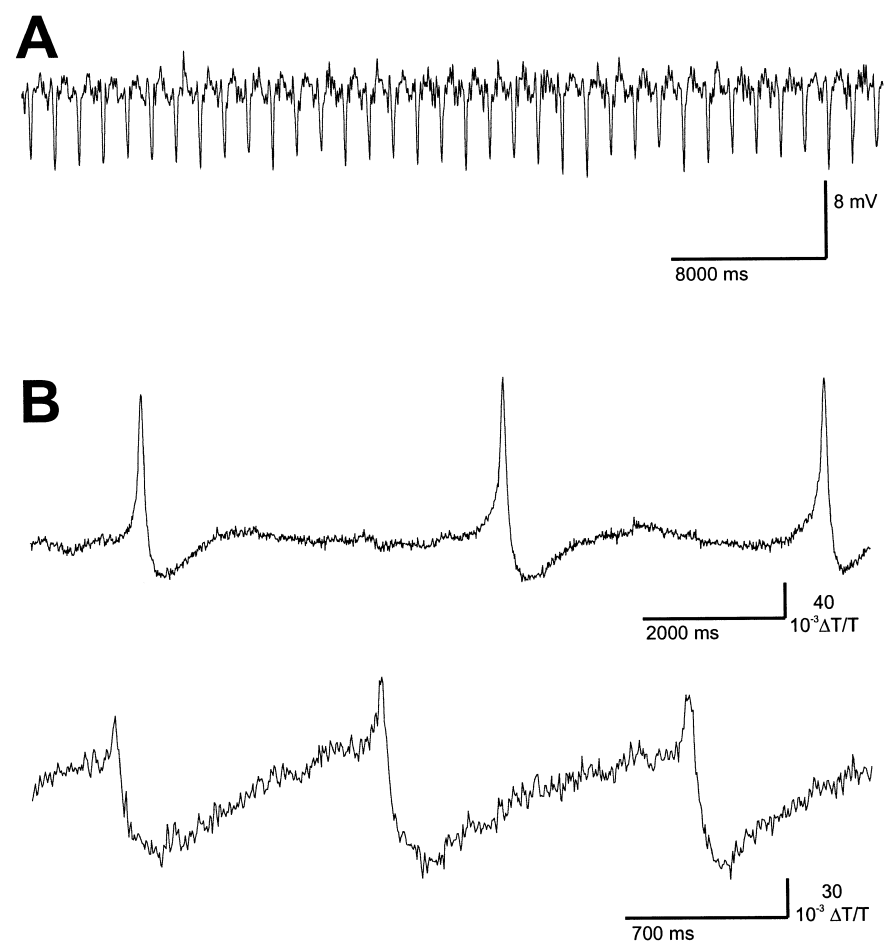

Figure 1 (A) Electrophysiological recording of procerebral oscillations in isolated CNS of Helix. (B) Optical recording of procerebral oscillations in snail Helix (top trace) and slug Deroceras (bottom trace). Averaging of the three adjacent channels. The wave of rhythmical activity displays the larger amplitude during ascending phase in Helix, while in Deroceras, the larger amplitude occurs during the descending phase. The amplitude was measured from the baseline.

izing peak amplitude to the hyperpolarizing amplitude (Fig. 1B). The oscillation shape in the PC of the slug Deroceras is very close to that in other slugs (Delaney et al. 1994; Kawahara et al. 1997). The wave shape was reproducible in all experiments using the optical recording method.

Other than spontaneous oscillations, we recorded odor-evoked waves of depolarization in the neuropile of the PC ( $n=46$ animals; Fig. 2). The evoked wave was not recorded in the cellular zone of the PC where the largest amplitude of spontaneous oscillations was observed (Fig. 2, traces from the cellular zone of the PC). The direction of propagation was determined by analyzing the shift of amplitude peak of the EP at each pixel of the photodiode array (Fig. 3). In the area accessible for optical recording, the EP originated in the olfactory nerve and propagated via the neuropile toward the cell body layer at a velocity of $30 \pm 10$ $\mathrm{mm} / \mathrm{sec}$ (Figs. 4, 5, and 6).

The odor-evoked potential recorded in the PC of Helix was never observed in six control experiments in which the olfactory epithelium was stimulated by a clean air puff from the olfactometer (data not shown). A relatively weak odor (5\%) elicited clear evoked potentials only in areas close to the place of the olfactory nerve entrance into the PC (18

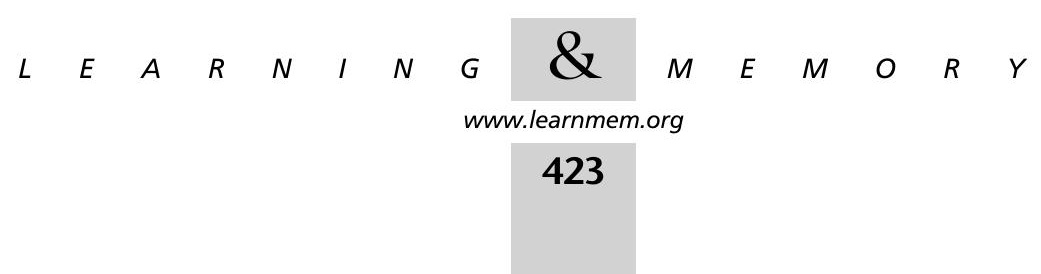




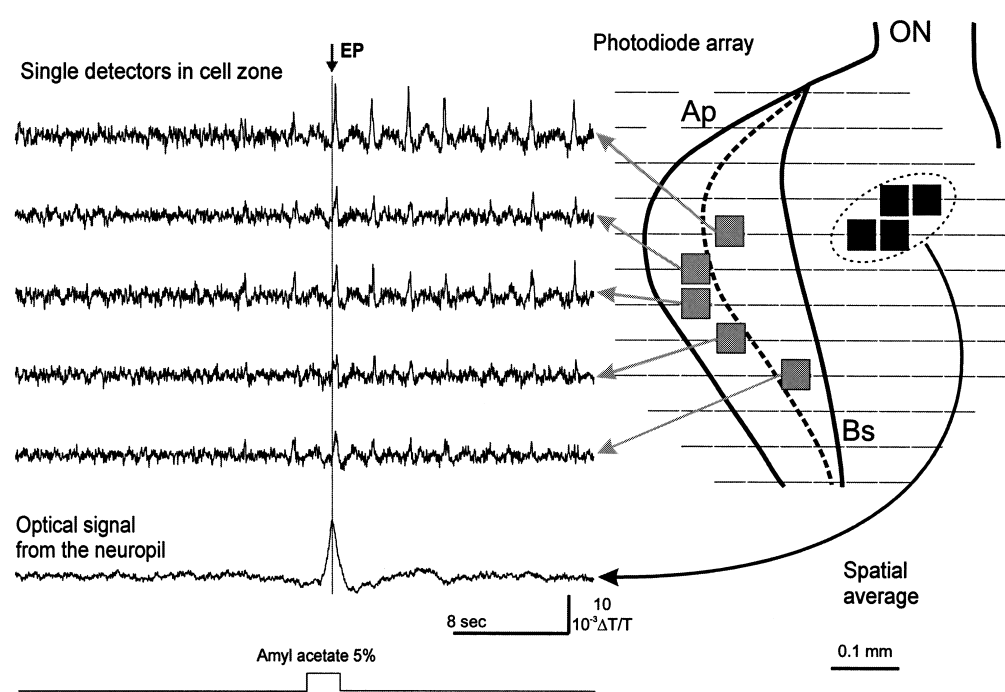

Figure 2 Scheme of the optical data representation. On the right, the procerebral contour is superimposed on the draft of a photodetector array. Each detector is represented by a line. The light passes through the preparation from the ventral side toward the dorsal one. Thus, the photodetector traces reflect the two-dimensional frontal projection of the electrical three-dimensional events in the PC. ON, olfactory nerve; Ap, apex of the PC; Bs, basal part of the PC; EP, odor-evoked potential. The gray boxes denote several photodiodes from the cell layer-area of spontaneous oscillations; the black boxes denote photodiodes from the neuropile. The traces from the area of oscillations are filtered with software at a bandpass of $0.6-19 \mathrm{~Hz}$, whereas the photodiode traces from the neuropile are filtered at a bandpass of $0.1-19 \mathrm{~Hz}$ and averaged.

experiments). A strong odor (20\%, 16 experiments) elicited potentials that were recorded over the entire neuropile projection to the plane of the photodiode array (Figs. 4A,C,D). The area of the spreading of the EP was mostly in the neuropile area and only partially overlapped with the area of spontaneous oscillations (Fig. 4B,C,D). In the example illustrated in Figure 4C, it can clearly be seen that the sponta-
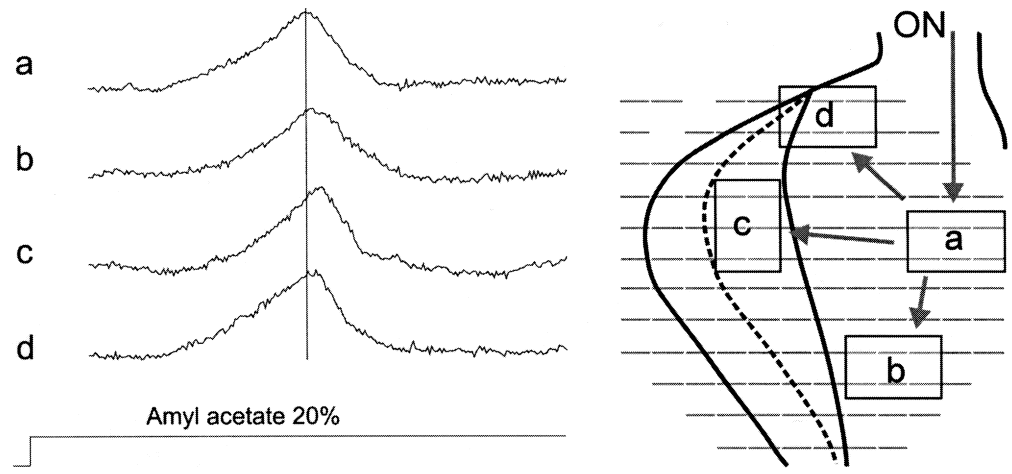

$$
200 \mathrm{~ms}=\begin{gathered}
80 \\
10^{+} \Delta \mathrm{T} / \mathrm{T}
\end{gathered}
$$

Figure 3 Spread of the odor-evoked potential. (Left), a shift of the EP peak amplitude timing synchronously optically recorded four areas of the PC under stimulation by $20 \%$ isoamyl acetate. The traces are averaged over the areas marked on the inset at right. The lettering of the wave corresponds to the area notation. (Right), scheme of the odor-evoked activity movement in PC. The arrows indicate the spreading direction. The wave appears first in the area a, then extends toward the other areas. neous oscillations do not spread into the neuropile, whereas the EP is spreading from the central neuropile to the border of the cell layer.

\section{Consistency in Responses to Odor}

The response to odor application consisted of two components: the EP (reflecting mostly the signal incoming to the PC) and changes in frequency of spontaneous oscillations, which may reflect the quality of odor. Applications of the same odor stimuli evoked consistent changes in spontaneous oscillation pattern (decrease or increase of frequency) in the same experiment. We observed several times that the responses to the same odors were different in different preparations (Fig 5, cf. A,B). We have not analyzed whether these differences depend on variability in responses or on the duration of stimulus and/or concentration of odor.

The presentation of odors with a small intertrial interval ( $3 \mathrm{~min}$ ) elicited a consistent and significant increase in latency of the EP (Fig. 6). On average, the increase in latency of the second response with a 3-min interval was $1.6 \pm 0.3 \mathrm{sec}$ (mean $\pm \mathrm{SEM}, n=12$ preparations, two-tailed $t$-test, $P<0.01)$. A control application of another odor elicited an EP with a small latency (Fig. 6, lower trace), suggesting that the observed increase in latency may be caused by some adaptation process specific to the presented odor.

Presentation of another odor also altered the direction of changes in amplitude of spontaneous oscillations in many cases (nine out of 12). In the example shown in Figure 7 , the odors were applied at a 3-min interval: twice isoamylacetate and in between, cineole. The response to the second application of isoamylacetate is evidently different from the response to the first one (decrease of amplitude instead of increase; Fig. 7). However, we did not perform any further analysis of this kind of short-term response change to different odors.

In an attempt to estimate the consistency of responses by comparing responses to same odor concentrations in different experiments, we compared only the first response to the given odor in a particular experiment. It was found in 16 of 18 experiments that the first responses to application of $5 \%$ cineole showed an increase in frequency of the spontaneous oscillations relative to the prestimulus level, and a decrease in frequency was noted only in two cases. In seven of 10 experiments, an application of $5 \%$ isoamylacetate elicited a decrease in frequency of spontaneous oscilla-

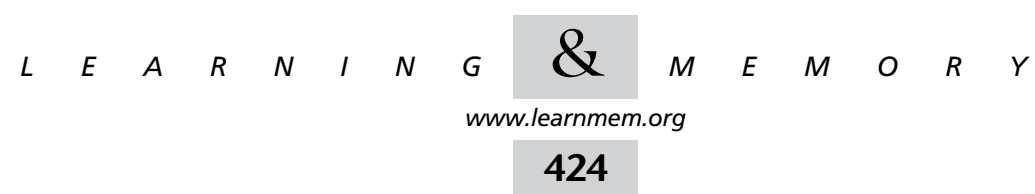



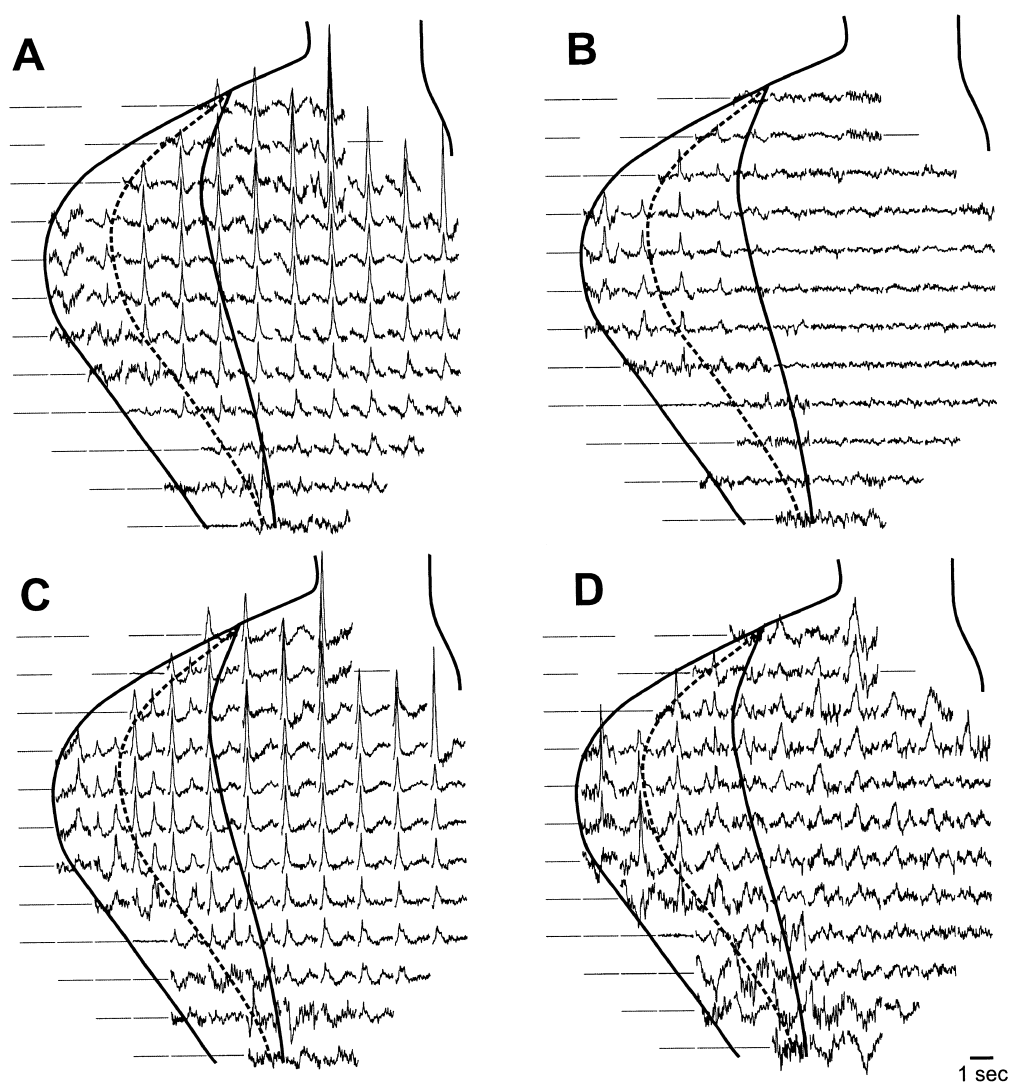

Figure 4 Distribution of odor-evoked potential and oscillatory wave in the PC (preparation of CNS with olfactory nerve and nose). The procerebral contour is superimposed on the draft of the photodiode array. Each detector is represented by a line. The traces of recordings are shown on correspondent detectors. (A) Spreading area of the potential evoked by application of $20 \%$ amyl acetate. (B) Spreading area of spontaneous oscillations, no odor applied. (C) Distribution areas of a spontaneous oscillation and the potential evoked by $20 \%$ amyl acetate. (D) Spreading areas of close in-time oscillation and potential evoked by 5\% amyl acetate. The evoked potential can be distinguished from the spontaneous oscillation by maximal amplitude at the place of entrance of the olfactory nerve (ON). Solid lines demarcate the procerebral contour and the area of oscillations; the broken line demarcates the odor-evoked potential spread.

tions, and an increase was seen only in three cases. This result suggests some consistency in responses to odors. Differences in responses to cineole and isoamyl acetate may be attributed to possible differences in behavioral significance of these odors.

\section{Differences in Responses to Odor in Control and Odor-Aversion-Trained Snails}

\section{Open Field}

Placing the snails in an open-field situation without any odor revealed no significant preference of the side or the odor cineole (Fig. 8A, $n=16$, Mann-Whitney rank sum test, $P=0.58)$. In some cases, the snails approached the odor source (with a $100 \%$ concentration of cineole in the vial) closer then $1 \mathrm{~cm}$. On average, in open-field experiments it was shown that snails do not distinguish between the situations with odor (cineole) and without it (Fig. 8A, $n=16$, M.W., $P=0.40$ ), suggesting behavioral indifference to cineole. Therefore, cineole was chosen as a stimulus for aversive training.

\section{Aversive Training}

The result of aversive conditioning was quantified by comparing the tentacle-withdrawal responses to $5 \%$ cineole application (duration 5 sec) in each snail before and after 16-20 d of training. Snails from experimental (paired presentations of odor and electric shock) and control (explicitly unpaired presentations of same stimuli) groups were tested in parallel using two similar setups. Double-blind procedure was used for behavioral testing. We used an estimate of tentacle (ommatophore) withdrawal in percentages of the maximal possible withdrawal taken as $100 \%$ (for details, see Balaban and Bravarenko 1993). Smallest detectable tentacle withdrawals in response to odor were estimated as 5\% responses. In each group, we were able to obtain electrophysiological results only from eight snails. Therefore, behavioral results were taken only for these particular snails. Averaged results showed that before training there was no significant difference in tentacle response amplitudes between the groups (Fig. 8B). After training, the median value of tentacle withdrawal in the experimental group increased to $56 \%$, whereas in the control group, which received the same stimuli in explicitly unpaired fashion, it stayed at the same low level (Fig. 8B, eight trained, eight control snails, Mann-Whitney rank sum test, $P<0.001)$.

Activity was recorded from the PC ipsilateral to the side of the body to which the electric shocks were applied in both the control and trained snails. We analyzed differences in changes induced by cineole application in frequency, amplitude, and proximal-to-distal ratio of the spontaneous oscillations. An example of the calculation of changes in response to odor application is shown in Figure 9. We calculated the amplitude as the difference between the top extreme and the bottom extreme of oscillations in each selected detector (Fig. 9A) and plotted it in a histogram (Fig. 9B,C). On two examples of recordings from the control (explicitly unpaired) group (Fig. 9B), and from the experimental (paired) group (Fig. 9C), it can clearly be seen that in spite of very similar changes in amplitude of oscillations, the proximal-to-distal ratio is quite different in those animals.

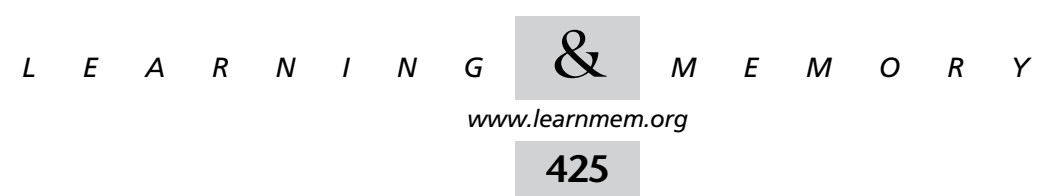



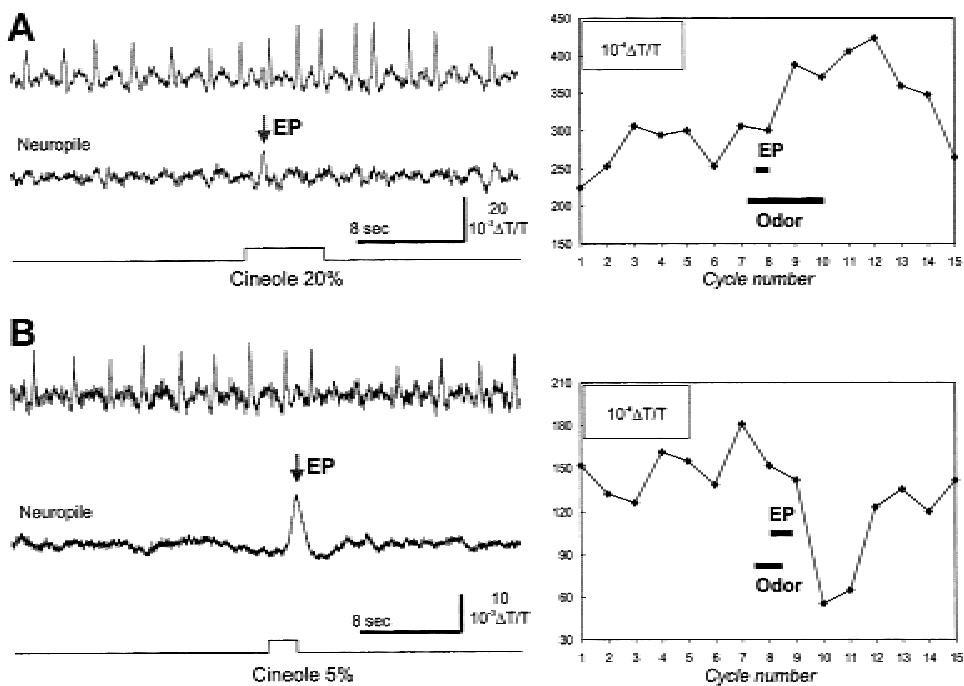

Figure 5 Changes in procerebral oscillations induced by odor applications in two animals. Upper trace on $(A)$ and $(B)$, representative recordings from one of the photodetectors on which a PC cell layer is projected. Bottom trace, odor-evoked potential (EP) recorded in the neuropile (inset). The changes in amplitude of the oscillations shown at the left, evoked by odor, are presented.

Averaged results showed that cineole application elicits an immediate significant increase in frequency of spontaneous oscillations relative to the prestimulation period (Fig. 10, upper panel), which is undistinguishable in trained and control animals (frequency values were calculated and

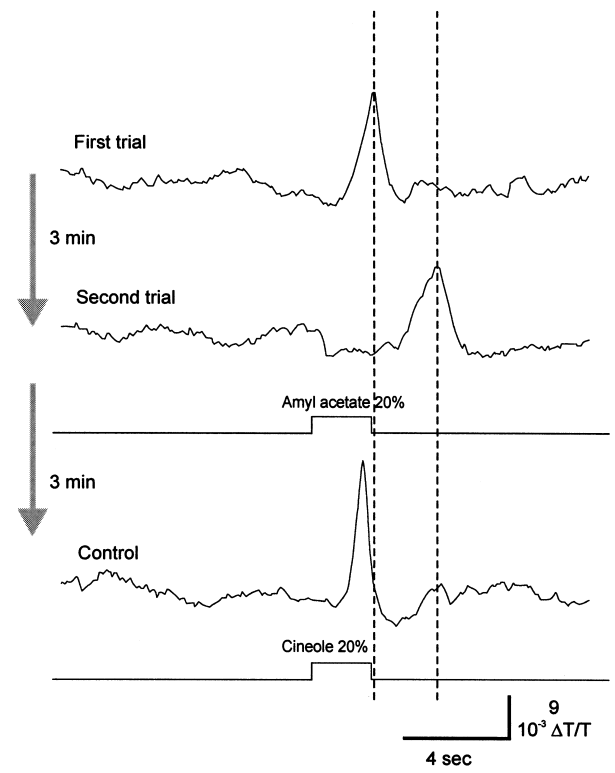

Figure 6 Changes in latency of the EP recorded in the neuropile. Three consequent responses to the repeated odor presentations are shown. Second presentation of the odor (IAA) with a 3-min interval elicited the EP with increased latency. Consequent presentation of another odor (cineole) with same 3-min interval confirmed that the preparation condition is good. compared in each 5 -sec bin; average frequency for $40 \mathrm{sec}$ before and $40 \mathrm{sec}$ after the beginning of odor presentation was compared; also, $n=8$, $P=0.78$ ). No significant changes were observed in averaged amplitudes of oscillations before and after odor application in trained and control snails (Fig. 10, middle panel, $n=8, P=0.82$ ). Significant differences between response parameters in trained and control snails were observed only in a proximal-to-distal ratio of spontaneous oscillations amplitudes (Fig. 10, bottom panel). A significant difference in proximal-to-distal ratio was noted at the intervals $10-15$ and $15-20 \mathrm{sec}$ after the beginning of odor presentation (Fig. 10, bottom panel, $n=8, \quad P<0.05)$. Comparison of averaged ratios after the beginning of odor presentation with the values of ratios before odor stimulation revealed that in trained animals the proximalto-distal ratios were significantly different from ratio before the odor at intervals 5-10, 10-15, and 15-20 sec (Fig. 10, bottom panel; Dunn's test, $P<0.05)$. Increase of the proximal-to-distal ratio suggests that amplitudes of oscillations after cineole presentation become relatively bigger in the proximal area of the PC, implying greater changes in neuronal firing in this area.

\section{DISCUSSION}

Evoked Potential and Spontaneous Oscillations The observed frequency of background oscillations in the Helix PC $(0.3-0.8 \mathrm{~Hz})$ is close to that reported for the slug Limax (0.69 Hz; Gelperin and Tank 1990). Moreover, our results demonstrated that the oscillations in Helix PC were waves that travel from the procerebral apex toward the basis. This is consistent with reported data on procerebral oscillations in Limax (Gelperin 1989). The calculated propagation velocity in our experiments $(3 \mathrm{~mm} / \mathrm{sec})$ is three times faster than that reported for Limax $(1.1 \mathrm{~mm}$ / sec; Delaney et al. 1994).

The spontaneous oscillations have both ascending and descending phases, the ratio of phases being different in the two species tested (Fig. 1B). The wave recorded in PC of Deroceras has a shape similar to that in Limax (Gelperin and Tank 1990; Kawahara et al. 1997), which was recorded with the fluorescent voltage-sensitive probe di-4-A-NEPPS. Responses to odor application in Helix are similar to odorelicited transient changes in oscillatory activity as reported for Limax (Gervais et al. 1996). These consistencies suggest some generality of olfactory coding in the two groups of terrestrial mollusks.

To interpret the physiological data, an understanding of anatomy and physiology of the PC is necessary. The ol-

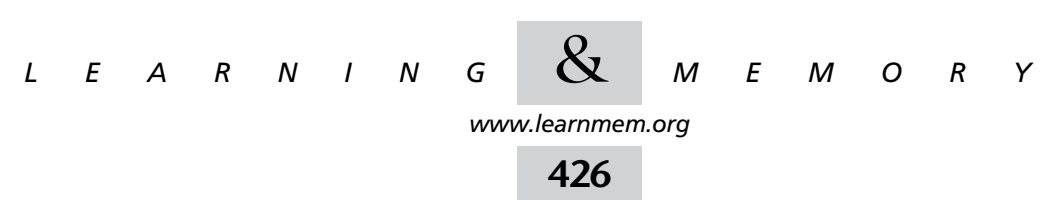




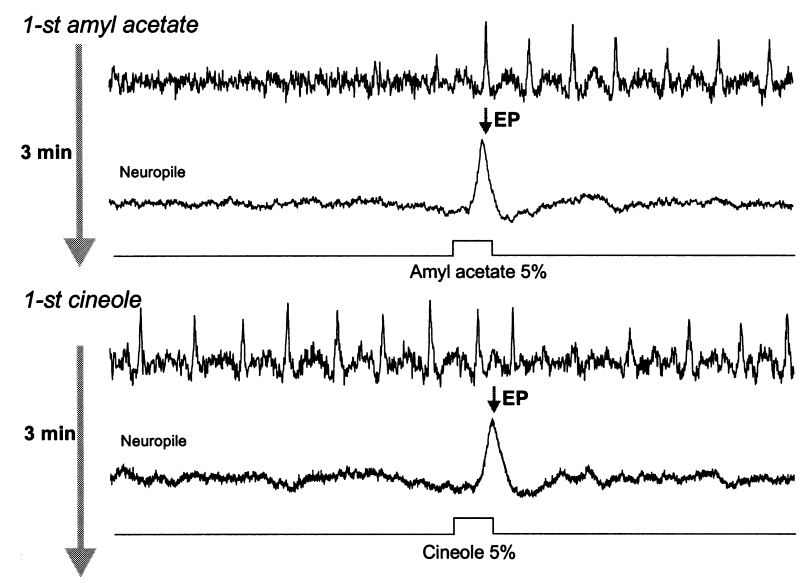

2-nd amyl acetate
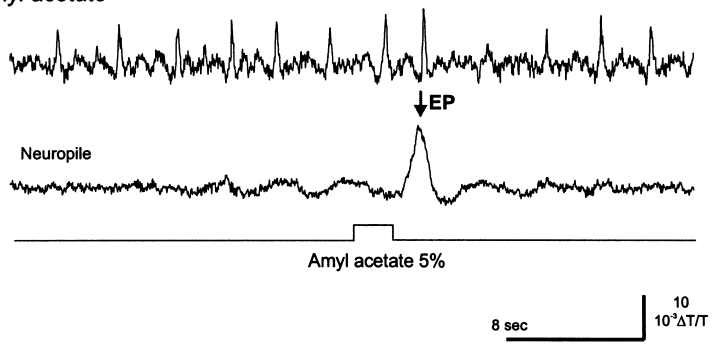

Figure 7 Application of another odor (cineole) changes the response to IAA. Successive recordings with 3 -min intervals are shown.

factory nerve enters the PC near its apical end. The nerve contains axons of sensory neurons as well as the axons of tentacular ganglion projection interneurons (Chase and Tolloczko 1993). The nerve backfills demonstrate that the majority of olfactory axons branch and terminate in the PC (Zaitseva 1991; Chase and Tolloczko 1993). The synaptic connections of those neurons occur both in the neuropile and in the cell body layer, as shown by intracellular staining of individual procerebral neurons (Ratté and Chase 1997). Moreover, the electron microscopic data suggested the presence of axosomatic synapses on cell bodies of the PC (Zs-Nagy and Sakharov 1970).

The PC contains two classes of neurons whose activity is phase-locked to the oscillations (Delaney et al. 1994). The $B$ cells have their somata and all their neurites and synapses in the cell layer of the PC (Watanabe et al. 1998). In contrast, the NB cells have somata in the cell layer, but their extensively branched neurites and synaptic connections are in the neuropile layer, where terminals of both sensory and presumed output neurons exist (Ratté and Chase 1997). Bursting (B) cells give brief bursts of spikes in the initial phase of the oscillation. Nonbursting (NB) cells receive a 5-7-mV IPSPs a few milliseconds after the bursts in B cells. The most apical B cells have the shortest burst period. As B cells are coupled to one another, the most apical B cells trigger the activity in the whole oscillating network. NB cells make up at least $90 \%$ of the cells in the PC (Delaney et al. 1994). This type of cells is thought to be associated with odor recognition (Ermentrout et al. 1998).

The odor-evoked potential (EP) recorded in our experiments in Helix originated in the olfactory nerve and spread to the neuropile. The EP only partially overlapped the area with maximal amplitude of spontaneous oscillations. We think that the use of absorption dyes, which allowed us to record from the neurites and nerves better than the fluorescent dyes (recording from the surface), explain why the EP to odors was not described before. Generation of the EP and spontaneous oscillations seems to be independent, without any occlusion (Fig. 4C,D).

The oscillatory waves in Helix propagate in the cell body layer from the procerebral apex toward the proximal part, whereas the EP originates near the olfactory nerve entrance into the PC and irradiates via the neuropile toward the cell body layer (Fig. 4C). The direction of the odor-evoked potential spreading is nearly orthogonal to the direction of oscillatory wave propagation. It is known that the somata layer in PC surrounds the neuropile on the ventral and dorsal sides. Our results indicate that the observed spreading of the EP to the area of oscillations may be a result of the overlapping of the neuropile projection (the part of PC where the EP has been recorded) and the projection of the cell body layer where the oscillations are most expressed (Delaney et al. 1994) to the two-dimensional plane of the photodiode array. This interpretation is in accord with the olfactory nerve backfill data (Zaitseva 1991; Chase and Tolloczko 1993). These authors demonstrated that the olfactory nerve fibers do not reach the cell body layer: They terminate in the procerebral neuropile, at the border of the cell body layer and the neuropile.

The EP was not recorded in the cell layer in our experiments. This suggests that the incoming activity via the olfactory nerve does not evoke a cyclic activity. This is confirmed by the fact that the odor-evoked potential never produced a new phase-independent oscillatory wave or a phase shift.

There are not enough data to conclude whether there is any spatial organization in the PC responses to odors. It would be necessary to examine more than two odorants in detail to reveal the presence or absence of a spatial organization. In our experiments, the PC responses to odors never showed spatial organization. This is consistent with the autoradiography mapping of neuropile and cell body layer using $\left[{ }^{14} \mathrm{C}\right]$-2-deoxyglucose label (Chase 1985). The data reported by Zakharov et al. (1998) demonstrated that the majority of PC neurons $(-70 \%-80 \%)$ appears during the first

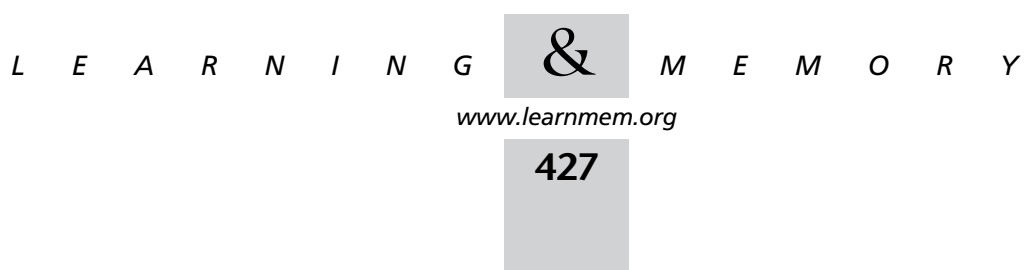



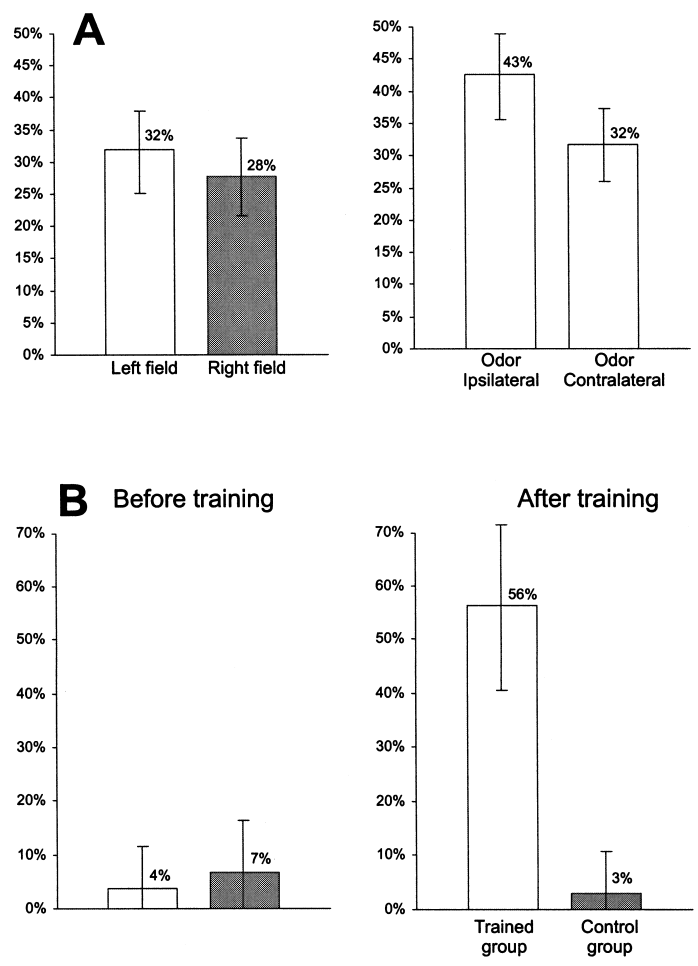

Figure 8 Averaged results of behavioral testing and training. (A) Percentage of the time the snails ( 16 snails, mean \pm SEM values are shown) spent in the left and right parts of the open field without odor (left plot) and with odor (cineole) located alternatively at the right and left field (right plot). Time while the snail's foot was at the middle line was not taken into account. (B) Percentage of the maximal tentacle contraction (complete contraction taken as $100 \%$ ) in response to $5 \%$ cineole application before (left panel) and after (right panel) 16 pairings (one per day) of cineole presentation with electric shock (eight snails, mean \pm SEM values are shown).

1-2 mo of posthatching development, displacing the previously produced neurons toward the basal part of the PC. At the same age, the snails display normal feeding behavior and an ability to learn about food odors (Croll and Chase 1980). Thus, the snails may be able to perceive the odor with a small portion of the PC that has not been formed completely. These results can be interpreted either way, that there is no spatial organization or that spatial organization can exist early on and then expand as the animal grows. Opposing the view that spatial organization does not exist in the PC lobe are the results obtained in trained animals. In these experiments, loci of plasticity were found in Limax (Kimura et al. 1998a), and our results are compatible with reported data.

\section{Changes in Responses Caused by Aversive Conditioning}

Behavioral experiments demonstrated that snails acquired a conditioned aversion to cineole applications after pairing sessions (Fig. 8B). We compared the responses of snails that received paired presentations of odor and electric shock with responses of animals that received explicitly unpaired presentations of the same stimuli (Balaban and Bravarenko 1993). Optically recorded changes in parameters of spontaneous oscillations in aversively trained and control snails were compared to find parameters related to learning. Comparison of responses in animals of the experimental group with those of the control group showed that only one parameter is consistently different, namely, proximal-to-distal ratio of spontaneous oscillation amplitudes. Responses to the trained odor were not different in amplitude or frequency of spontaneous oscillations to control and experimental trained snails (Fig. 10). It is important to note that maximal increase of the proximal-to-distal ratio was observed 10-15 sec after the stimulus presentation (Fig. 10). We know from behavioral experiments that response latency is $11 \pm 4 \mathrm{sec}$. This suggests that changes in the proximal-to-distal ratio precede and may reflect the behavioral changes elicited by training. Additional experiments are needed to understand whether these changes are specific for aversive training.

Interestingly, changes in uptake of Lucifer Yellow immediately after aversive learning to odors were shown in similar (proximal) region of the PC of Limax (Kimura et al. 1998a; Teyke et al. 2000). The authors suggest that enhancement of neural activity occurred in labeled regions and speculate that this region may be related to the formation of odor memory. The observed changes in the pattern of spontaneous oscillations in our experiments correspond to the same location (Kimura et al. 1998c). Spontaneous oscillations frequency changes to trained odor application in Helix do not correspond to pairing-specific changes in oscillations frequency observed in Limax (Kimura et al. 1998b), which may be attributed to differences in training procedure. Recently, it was shown that reversible blockage of the oscillations did not impair recognition of conditioned odors but reduced the differential nerve response to related odors (Teyke and Gelperin 1999).

Local increases of amplitude of the spontaneous oscillations are usually interpreted as indicating an increase of synchronization of neural firing in that particular locus (Delaney et al. 1994) in our case, for example, the olfactory lobe basal area. It can be suggested that such synchronization is caused by activation of the NB-cells, which were shown to be hyperpolarized and normally silent without the odor (Ermentrout et al. 1998). Sometimes the NB cells produce irregular spikes in phase of the oscillation (Delaney et al. 1994).

Finally, in our experiments, the odor produced a relatively fast evoked potential that appeared mostly in the neuropile of the PC (Fig. 4) and the long-lasting changes of the oscillation parameters. Only one of the recorded parameters (frequency and amplitude of the spontaneous oscillations, proximal-to-distal ratio) of the PC responses to odors, the proximal-to-distal ratio, was shown to be pairing specific and to reflect the local changes in neural firing elicited by the training procedure.

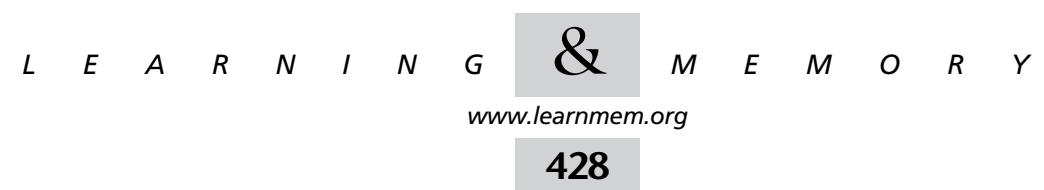




\section{MATERIALS AND METHODS}

\section{Preparation}

The experiments were performed on isolated CNS and semi-intact preparations with intact olfactory nerve and nose (Fig. 11) of the snail Helix lucorum L. obtained from the Crimea. Approximately 90 adult snails were used in the experiments. Several recordings

A

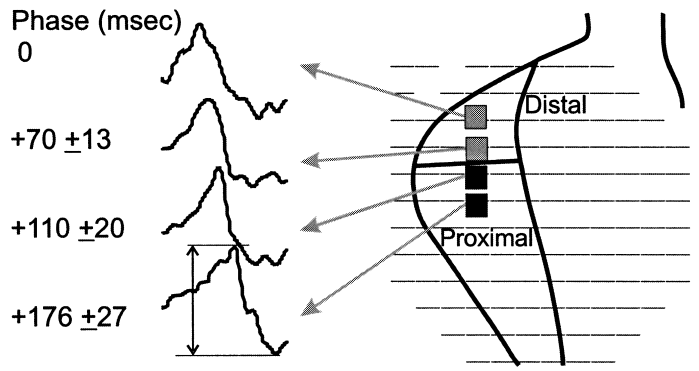

B Distal
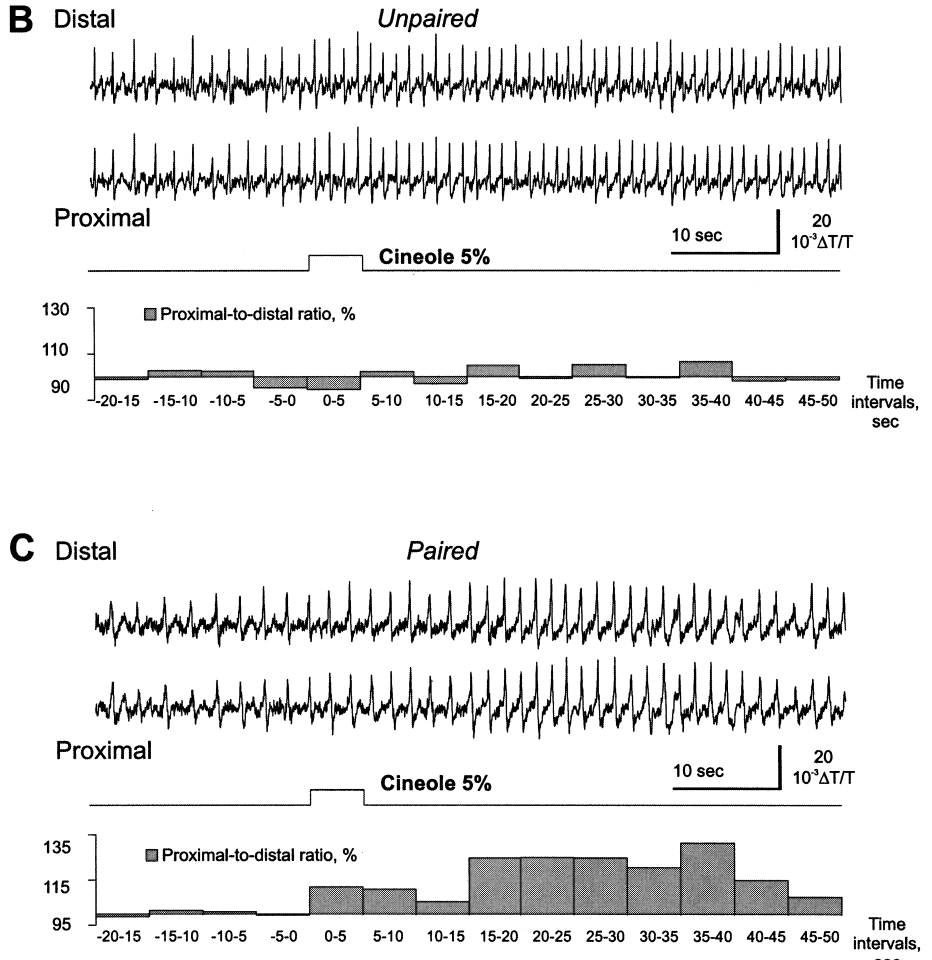

Figure 9 Changes in the proximal-to-distal ratio (the ratio of the spontaneous oscillation amplitude in the proximal part of the PC to the oscillation amplitude in the distal part) in response to odor application. (A) Representative recordings from detectors shown on the PC image inset and principle of amplitude calculation. Figures at left show mean peak latency \pm SEM for each oscillation before the odor. Earliest (usually most distal) peak was taken as having a zero latency. We calculated the amplitude as the difference between the top extremum and the bottom extremum of the oscillation. (B) Representative traces (distal and proximal) from the experiment in which there were no changes in the proximal-to-distal ratio; control group of snails can be seen on the accompanying histogram. (C) Representative traces from experiment in which there were notable changes in the proximal-to-distal ratio; experimental group of snails can be seen on the accompanying histogram. were also made in isolated CNS of the slug Deroceras reticulatum Müll (laboratory reared). Before dissection, the animals were anesthetized by injection of an isotonic solution of $\mathrm{MgCl}_{2}$ and by cooling. The Helix saline solution composition was as follows (concentration in $\mathrm{mM}$ ): $100 \mathrm{Na}^{+}, 4.2 \mathrm{~K}^{+}, 7.0 \mathrm{Ca}^{2+}, 4.6 \mathrm{Mg}^{2+}, 127.4 \mathrm{Cl}^{-}, 0.2 \mathrm{H}_{2} \mathrm{PO}_{4}^{-}$, $2.5 \mathrm{HCO}_{3}^{-}, 5.0$ glucose, $10 \mathrm{~N}$-2-hydroxyethylpyrasine-N'-2-ethanesulfonic acid (HEPES) at pH 7.6 (modified from Rhines et al. 1993).

The PC lobe was desheathed completely or partially, using fine forceps. After removal of the connective tissue from the surface of the PC, the inner layer of sheath was treated by protease (type IV, Sigma; $2.5 \mathrm{mg} / \mathrm{mL}$ ) for $10 \mathrm{~min}$ and, in some cases removed. During treatment, the olfactory epithelium (nose) was above the saline level and was not affected by protease. In case of partial desheathing, the inner layer was kept intact. Most of the presented results were obtained in preparations that were partially desheathed.

\section{Recording}

We recorded the PC activity electrophysiologically and optically. Electrophysiological recordings were performed using suction glass electrodes (inner diameter $\sim 50 \mu \mathrm{m}$ ). The recorded potentials were fed to a microelectrode amplifier set at a bandpass of $0.1-50 \mathrm{~Hz}$. The amplifier output was digitized and the result stored in an IBM-compatible computer.

The optical recording was performed with fast-response probe RH-155 (NK-3041), a member of the pyrazooxonol voltage-sensitive dye group. The protocol of staining was as follows: $2 \times 2 \mathrm{~min}$ of staining with saline containing RH-155 at a concentration of $0.2 \mathrm{mg} / \mathrm{mL}$, with a $4-\mathrm{min}$ interval and washout between staining. Recording was performed using transmitted light and 124 photodiodes with individual amplifiers (details in Cohen 1988. The wavelength of the filter used was $710 \pm 20 \mathrm{~nm}$. The filtered light passed through the preparation and was then projected via the optical system of a microscope on the photodiode array (124 detectors, Physical-Technical Institute). The microscope was equipped with a contact fluorescent objective $(\times 25,0.4 \mathrm{NA}, \mathrm{LOMO})$. The amplifier outputs were digitized with a 12-bit analog-to-digital converter (Microstar laboratories DAPL $3000 \mathrm{~A}, 417 \mathrm{kHz}$ ) under control of a Pentiumbased computer. The $4.8 \mathrm{msec}$ temporal resolution of recording was set in the software. The measured optical signal reflected the change in light transmission relative to its mean value $(\Delta \mathrm{T} / \mathrm{T})$. Neuroplex (OptImaging LLC) software for optical data analysis was used. The calculation of the propagation velocity and the definition of wave-spreading direction were performed using a cross-correlation analysis.

\section{Odor Application}

We stimulated the olfactory epithelium with two chemicals, cineole (Sigma) and isoamyl acetate (IAA, Sigma). The odors were delivered to the nose by a computer-controlled olfactometer (modified from Kauer and Moulton 1974) that allows manipulations of odor concentration and stimulation time (Fig. 12). The odor concentration ranged within 5\%$100 \%$ of saturated concentration by changing the ratio of the air flow rate through the odor-containing chamber and that of clean air. Before application to the nose, the two flows were mixed. We measured the airflow with two flowmeters (GF-1100, Gilmont Instruments). The total flow was $100-$

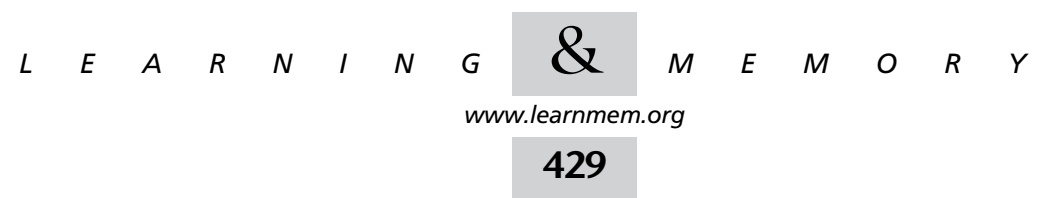




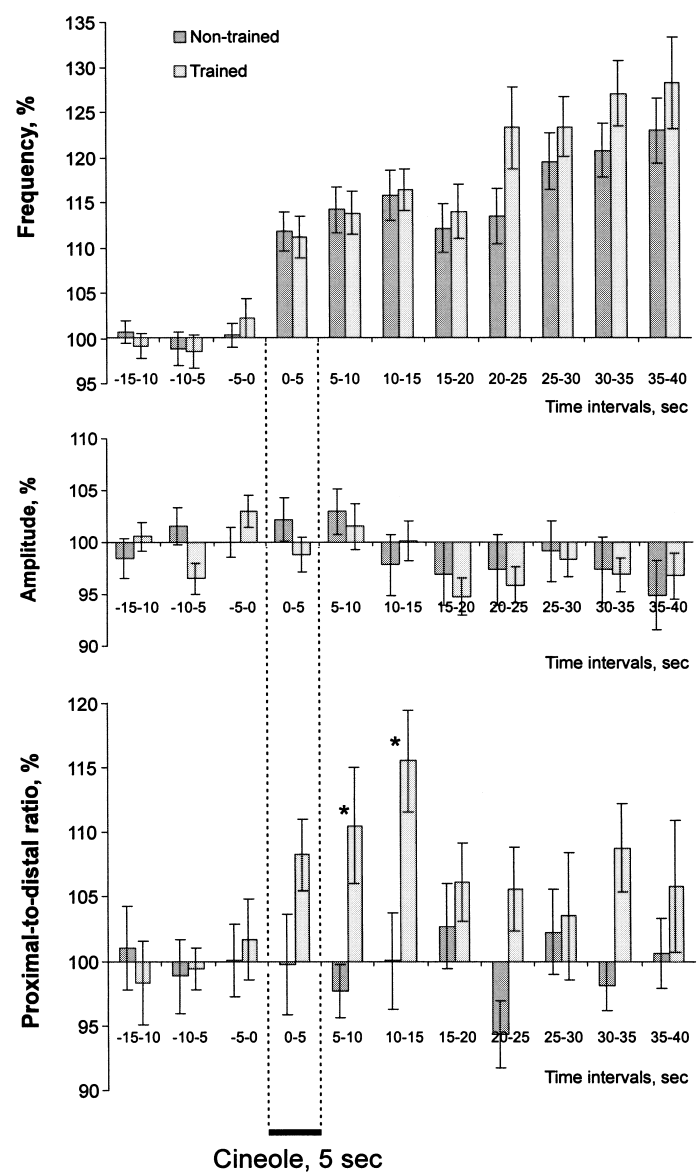

Figure 10 Comparison of changes induced by cineole application in frequency (upper histogram), amplitude (middle), and proximalto-distal ratio (bottom) of the spontaneous oscillations in the PC lobe of aversively trained and control snails.

$110 \mathrm{~mL} / \mathrm{min}$. The odor was constantly propelled from the olfactometer output tube positioned at $15 \mathrm{~mm}$ from the receptor surface and evacuated from the tip with suction. The rate of odor evacuation was 10 times higher than the rate of total flow. Electrically driven interruption of active odor removal resulted in odor spreading to the object (Fig. 12).

\section{Behavioral Experiments}

\section{Open Field}

To estimate an inborn negativity or attraction to the odor in the snails, we used an open-field test. A glass surface $60 \times 80 \mathrm{~cm}$ divided into $30 \times 40$ labeled squares was used. One snail was positioned at the beginning of experiment to the midline, and its position was traced each minute (Fig. 13A). The odor source (a vial with filter paper moistened with the odorant) was placed in the middle of the left or right side. Each snail was tested with and without odors, and odor position was alternated. The glass was washed after each snail, and the room was constantly ventilated. Maximal testing time in each trial was $10 \mathrm{~min}$. Percentage of time spent at the side with the tested odor served as an estimate of odor aversiveness or attractability of the respective odor

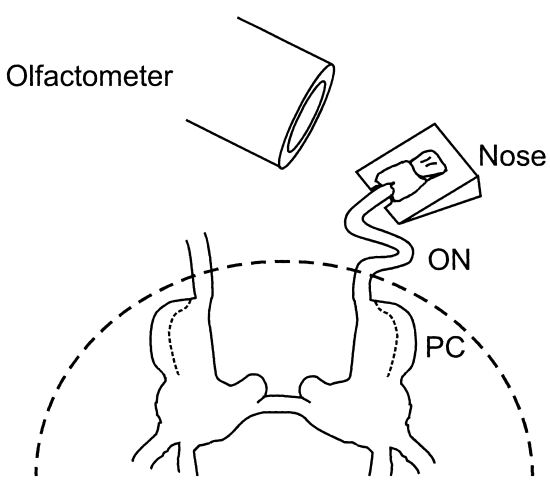

Figure 11 Scheme of preparation of CNS with attached intact olfactory nerve $(\mathrm{ON})$ and nose located on a small platform. The cerebral ganglia and the procerebral lobe (PC) are shown. Broken line shows the wall of a chamber with the CNS.

\section{Aversive Training}

To investigate the behavioral significance of odors, we used a conditioning protocol that was used previously in terrestrial snails for food-aversion conditioning (Balaban et al. 1987). The snail was tethered by its shell in a manner allowing it to crawl on a ball that rotated freely in a $0.01 \%$ solution of $\mathrm{NaCl}$ (Fig. 13B). Electric shocks $(110 \mathrm{~mA}, 0.5 \mathrm{sec}, 10 \mathrm{~Hz})$ were delivered through a macroelectrode applied manually to the right dorsal surface of the tail. The circuit was completed by contact of the foot with interlaced stainless steel wires on the surface of the ball, which in turn, contacted the grounded salt solution (Fig. 13B). The current intensity was adjusted for each snail such that it was just sufficient to cause com-

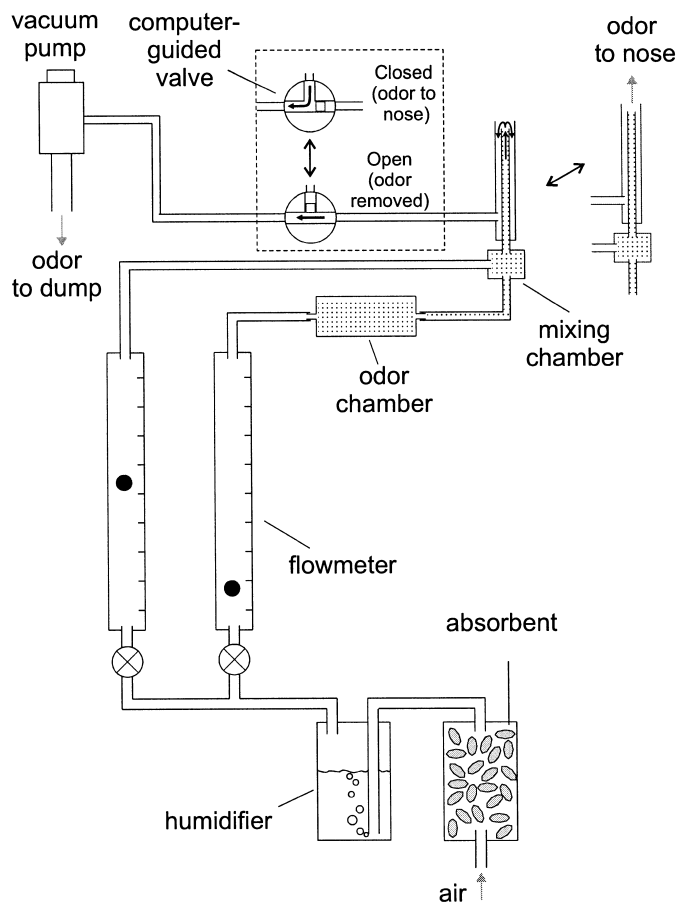

Figure 12 Scheme of the olfactometer used for odor application. The computer-controlled valve cancels the active odor evacuation and triggers the odor application.

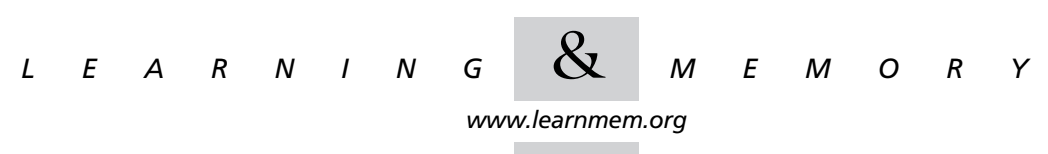



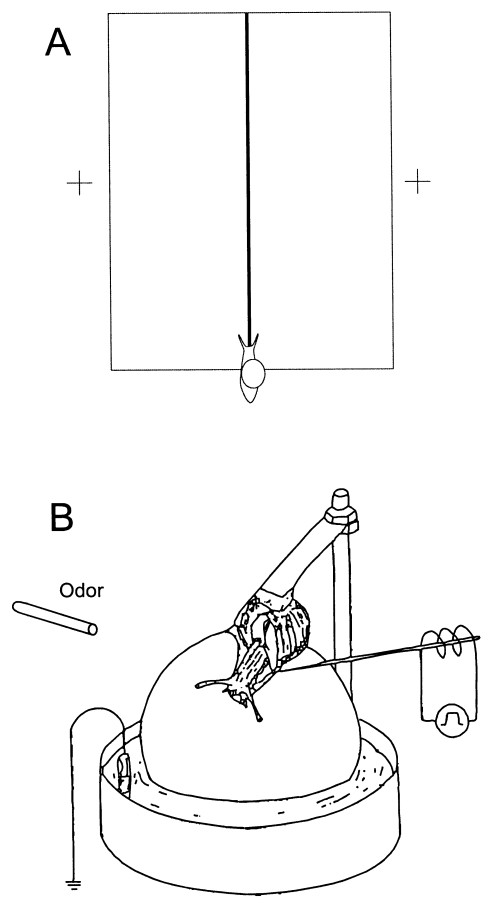

Figure 13 Scheme of the behavioral experiments. (A) Experimental arrangement for training snails to odor cues. (Odor). Output of the odor chamber located $15 \mathrm{~mm}$ from the snail's head. (B) Open field experiments (two locations of odor source are marked by crosses). The snail is shown in a start position.

plete withdrawal of the snail into the shell. Each session started with subthreshold stimulation, which was increased stepwise until complete withdrawal was elicited. This amplitude of stimulation was used for the particular snail in this session. No injury to the skin was observed after such electrical stimuli (necrosis is readily seen in snails because of changes in pigmentation and changes in mucus release). The odor source (cineole, the concentration at the location of the animal- $0.1 \%-2 \%$-was roughly estimated to be inversely proportional to the distance while passively diffusing from the $100 \%$ source) was placed at a $4-6-\mathrm{cm}$ distance from the snail's head. Electric shock (one paired trial per day) was delivered 10-15 sec after beginning the odor presentation. Interval between odor presentation and electric shock (one presentation of each stimulus per day) in explicitly unpaired control group ranged between 30 and $60 \mathrm{~min}$. The experimental (paired) and control (explicitly unpaired) groups consisted of 12 animals each. Training lasted for 16-20 d. During double-blind testing procedures, we estimated the maximal amplitude of tentacle withdrawal response in actively locomoting snails to the odor presentation lasting $30 \mathrm{sec}$. Testing and training were performed in a constantly ventilated room. After behavioral testing, we made preparations in which the responses to $5 \%$ cineole (delivered using the olfactometer from a distance of 15 $\mathrm{mm}$ ) were recorded optically from the PC.

\section{ACKNOWLEDGMENTS}

We thank Dr. A. Gelperin for constant interest and support, Dr. G. Cymbaluk for valuable suggestions, Dr. L.B. Cohen for helpful criticism, and Dr. N. Bravarenko for behavioral testing. This work was partly supported by grants from the Russian Foundation for Basic
Research, NATO grant HTECH.LG973297, and Howard Hughes Medical Institute grant 75195-544301.

The publication costs of this article were defrayed in part by payment of page charges. This article must therefore be hereby marked "advertisement" in accordance with 18 USC section 1734 solely to indicate this fact.

\section{REFERENCES}

Balaban, P.M. 1993. Behavioral neurobiology of learning in terrestrial snails. Prog. Neurobiol. 41: 1-19.

Balaban, P. and Bravarenko, N. 1993. Long-term sensitization and environmental conditioning in terrestrial snails. Exp. Brain Res. 96: $487-493$.

Balaban, P.M., Vehovszky, A., Maximova, O.A., Zakharov, I.S. 1987. Effect of 5,7-dihydroxytryptamine on the food-aversive conditioning in the snail Helix lucorum L. Brain Res. 404: 201-210

Chase, R. 1985. Responses to odors mapped in snail tentacle and brain by [14C]-2-deoxyglucose autoradiography. J. Neurosci. 5: 2930-2939.

Chase, R. and Tolloczko, B. 1993. Tracing neural pathways in snail olfaction: From the tip of the tentacles to the brain and beyond. Microsc. Res. Tech. 24: 214-230.

Cohen, L.B. 1988. More light on brains. Nature 331: 112-113.

Croll, R. and Chase, R. 1980. Plasticity of olfactory orientation to foods in snail Achatina fulica. J. Comp. Physiol. 136: 267-277.

Delaney, K.R., Gelperin, A., Fee, M.S., Flores, J.A., Gervais, R., Tank, D.W., and Kleinfield, D. 1994. Waves and stimulus-modulated dynamics in an oscillating olfactory network. Proc. Natl. Acad. Sci. 91: 669-673.

Ermentrout, B., Flores D., and Gelperin, A. 1998. Minimal model of oscillation and waves in the Limax olfactory lobe with tests of model's predictive power. J. Neurophysiol. 79: 2677-2689.

Friedrich, A. and Teyke, T. 1998 Identification of stimuli and input pathway mediating food-attraction conditioning in the snail, Helix. J. Comp. Physiol. 183: 247-254.

Gelperin, A. 1989. Neurons and networks for learning about odors. In Perspectives in neural systems and behavior, (eds. T.J. Carew and D.B. Kelly) pp. 121-136. Liss, New York.

Gelperin, A. and Tank, D.W. 1990. Odor-modulated collective network oscillations of olfactory interneurons in a terrestrial mollusk. Nature 345: 437-440.

Gervais, R., Kleinfield, D. Delaney, K.R. and Gelperin, A. 1996. Central and reflex neuronal responses elicited by odor in a terrestrial mollusk. $J$. Neurophysiol. 76: 1327-1339.

Gray, C.M. and Skinner, J.E. 1988. Centrifugal regulation of neuronal activity in the olfactory bulb of the walking rabbit as revealed by reversible cryogenic blockade. Exp. Brain. Res. 69: 378-386.

Kauer, J.S. and Moulton, D.G. 1974. Responses of olfactory bulb neurons to odor stimulation of small nasal areas in the salamander. J. Physiol. 243: 717-737.

Kawahara, S., Toda, S., Suzuki, Y., Watanabe, S., and Kirino, Y. 1997. Comparative study on neural oscillation in the PC of the terrestrial slugs Incilaria bilineata and Limax marginatus. J. Exp. Biol. 200: 1851-1861

Kimura, T., Suzuki, H., Kono, E., and Sekiguchi, T. 1998a. Mapping of interneurons that contribute to food aversive conditioning in the slug brain. Learn. Mem. 4: 376-388.

Kimura, T., Toda, S., Sekiguchi, T., Kawahara, S., and Kirino, Y. 1998b. Optical recording analysis of olfactory response of the procerebral lobe in the slug brain. Learn. Mem. 4: 389-400.

Kimura, T., Toda, S., Sekiguchi, T., and Kirino, Y. 1998c. Behaviora modulation induced by food odor aversive conditioning and its influence on the olfactory responses of an oscillatory brain network in the slug Limax marginatus. Learn. Mem. 4: 365-375.

Laurent, G. and Davidowitz, H. 1994. Encoding of olfactory information with oscillating neural assemblies. Science 265: 1872-1875.

Mellon Jr., D., Sandeman, D.S., and Sandeman, R.E. 1992. Characterization of oscillatory olfactory interneurons in the protocerebrum of the crayfish. J. Exp. Biol. 167: 15-38.

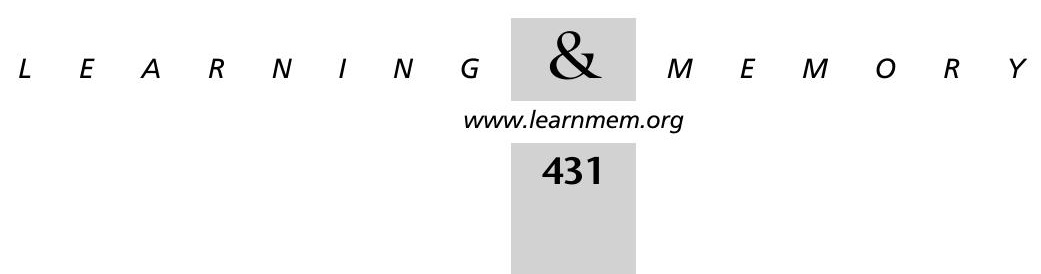


Ratté, S. and Chase, R. 1997. Morphology of interneurons in the PC of the snail Helix aspersa. J. Comp. Neurol. 384: 359-372.

Rhines, L.D., Sokolove, P.G., Flores, J., Tank, D.W., and Gelperin, A. 1993. Cultured olfactory interneurons from Limax maximus: Optical and electrophysiological studies of transmitter-evoked responses. $J$. Neurophysiol. 69: 1940-1947.

Sahley, C., Gelperin, A., and Rudy, J.W. 1981. One-trial associative learning modifies food preferences of a terrestrial mollusk. Proc. Natl. Acad. Sci. 79: 640-642.

Steinmetz, P.N., Roy, A., Fitzgerald, P.J., Hsiao, S.S., Johnson, K.O., and Niebur, E. 2000. Attention modulates synchronized neuronal firing in primate somatosensory cortex. Nature 404: 187-189.

Teyke, T. and Gelperin, A. 1999. Olfactory oscillations augment odor discrimination not odor identification by Limax. CNS NeuroReport 10: $1061-1068$.

Teyke, T., Wang, J.W., and Gelperin, A. 2000. Lateralized memory storage and crossed inhibition during odor processing by Limax. J. Comp. Physiol. 186: 269-278.
Watanabe, S., Kawahara, S., and Kirino, Y. 1998. Morphological characterization of the bursting and nonbursting neurons in the olfactory center of the terrestrial slug Limax marginatus. J. Exp. Biol. 201: 925-930,

Wehr, M. and Laurent, G. 1996. Odor encoding by temporal sequences of firing in oscillating neural assemblies. Nature 384: 162-166.

Zaitseva, O.V. 1991. Structural organization of the tentacular sensory system in land pulmonates. In Simpler nervous systems, (eds. D.A. Sakharov and W. Winlow) pp. 238-257. Manchester University Press, Manchester.

Zakharov, I.S., Hayes, N.L., Ierusalimsky, V.N, Novakowski, R.S., and Balaban, P.M. 1998. Postembryonic neurogenesis in the PC of the terrestrial snail Helix lucorum L. J. Neurobiol. 35: 271-276.

Zs-Nagy, I. and Sakharov, D.A. 1970. The fine structure of the PC of pulmonate mollusks, Helix and Limax. Tissue Cell 2: 399-411.

Received April 14, 2000; accepted in revised form September 18, 2000. 


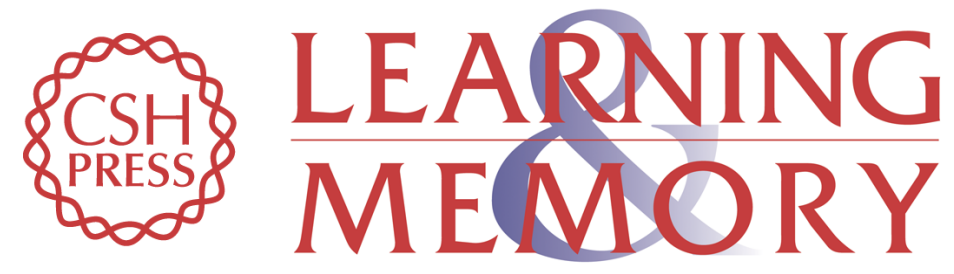

\section{Optical Recording of Odor-Evoked Responses in the Olfactory Brain of the Nai?ve and Aversively Trained Terrestrial Snails}

Eugeny S. Nikitin and Pavel M. Balaban

Learn. Mem. 2000, 7:

Access the most recent version at doi:10.1101//m.32500

References This article cites 29 articles, 9 of which can be accessed free at: http://learnmem.cshlp.org/content/7/6/422.full.html\#ref-list-1

License

Email Alerting Receive free email alerts when new articles cite this article - sign up in the box at the Service top right corner of the article or click here. 